\title{
Spine radiosurgery: lessons learned from the first 100 treatment sessions
}

\author{
Ran Harel, MD, ${ }^{1-3}$ Raphael Pfeffer, MBBS, ${ }^{4}$ Daphne Levin, PhD, ${ }^{4}$ Efrat Shekel, MA, ${ }^{4}$ \\ Dan Epstein, MSc, ${ }^{4}$ Lev Tsvang, MSc, ${ }^{5}$ Maoz Ben Ayun, MA, ${ }^{5}$ Dror Alezra, PhD, ${ }^{5}$ and \\ Leor Zach, MD ${ }^{3,5}$
}

1Stereotactic Radiosurgery Unit and ${ }^{2}$ Spine Surgery Unit, Talpiot Medical Leadership Program, Department of Neurosurgery, Sheba Medical Center, Ramat Gan; ${ }^{3}$ Sackler Medical School, Tel-Aviv University; ${ }^{4}$ Department of Radiotherapy, Assuta Medical Centers, Tel-Aviv; and ${ }^{5}$ Radiation Oncology Unit, Oncology Institute, Sheba Medical Center, Ramat Gan, Israel

OBJECTIVE Local therapy to spine tumors has been shown to be effective in selected cases. Spinal radiosurgery (SRS) is an evolving radiotherapy regimen allowing for noninvasive, highly efficacious local treatment. The learning curve can compromise the results of any newly employed technology and should be studied to minimize its effects. In this paper the first 100 SRSs performed at several medical centers are presented and analyzed for the effects of the learning curve on outcome.

METHODS A retrospective analysis was undertaken to evaluate data from patients treated with SRS at Sheba Medical Center and Assuta Medical Centers in the period from September 2011 to February 2016. Medical history, clinical and neurological findings, pathological diagnoses, SRS variables, complications, and follow-up data were collected and analyzed. Local control rates were calculated, and local treatment failure cases were qualitatively studied.

RESULTS One hundred treatment sessions were performed for 118 lesions at 179 spinal levels in 80 patients. The complication rate was low and did not correlate with a learning curve. Mean follow-up time was 302 days, and the overall local control rate was $95 \%$. The local control rate was dose dependent and increased from $87 \%$ (among 35 patients receiving a dose of $16 \mathrm{~Gy}$ ) to $97 \%$ (among 65 patients receiving a dose of $18 \mathrm{~Gy}$ ). The 6 treatment failure cases are discussed in detail.

CONCLUSIONS Spinal radiosurgery is a safe and effective treatment. Comprehensive education of the treating team and continuous communication are essential to limit the effects of the learning curve on outcome.

https://thejns.org/doi/abs/10.3171/2016.9.FOCUS16332

KEY WORDS spine tumors; spine radiosurgery; learning curve

$\mathrm{E}$ ARLY diagnosis and improved treatments and patient care steadily increase median survival rates in cancer patients. ${ }^{7,24}$ Extended survival of patients with metastases compels the treatment of bone metastases and specifically spine metastases. ${ }^{15,25}$ Patchell et al. ${ }^{23}$ changed the treatment algorithm for epidural tumors demonstrating evident benefit from surgery combined with radiotherapy. In alignment with the data in this publication, the rationale for local targeted therapy for patients with oligo- metastases and those with radioresistant tumors became widely accepted. Over the years, improved technological solutions have enabled cranial radiosurgery techniques to be transformed into radiosurgery for spinal tumors. ${ }^{4,5}$, ${ }^{10-13}$ Spinal radiosurgery (SRS) allows for targeted treatment of spinal tumors with a high local control rate, high pain control rate, and low complication rate. ${ }^{11,14}$ Once local treatment was shown to be effective for selected patients with metastases, SRS became an attractive alternative to

ABBREVIATIONS ATRT = atypical teratoid/rhabdoid tumor; $\mathrm{CBCT}=$ cone-beam CT; CTV = clinical target volume; Dmax = maximum cord point dose; IMRT = intensitymodulated radiotherapy; NST = nerve sheath tumor; SRS = spinal radiosurgery; VMAT = volumetric modulated arc therapy.

SUBMITTED August 23, 2016. ACCEPTED September 12, 2016.

INCLUDE WHEN CITING DOI: 10.3171/2016.9.FOCUS16332. 
surgery. Spinal radiosurgery is also used as an adjunct to surgery, improving local control rates after surgical decompression, reducing the need for large resections and thus limiting surgical morbidity, and lowering the risk of wound-related complications and instrumentation failure. ${ }^{16,17}$

A learning curve is expected in any new medical procedure. The learning curve is often defined as the time after which a procedure can be performed safely and with a plateau in efficiency, usually correlated to the operative time. Spinal radiosurgery requires the cooperation of a neurosurgeon, radiation oncologist, neuro-radiologist, medical physicist, imaging technicians, radiation technicians, and nurses. Thus, the procedure is prone to a long and shallow learning curve that can result in low efficacy rates and high complication rates. The SRS technology is constantly changing, providing improved treatment planning and delivery but also extending the learning curve. A steep learning curve can be achieved by dedicated personnel with excellent training who are striving for excellence, obligated to team work, and equipped with good communication skills and workflow guidelines including regular meetings (that is, a tumor board). Since the critical components of SRS are performed before the actual treatment, they can be manipulated, checked, and rechecked until perfected, so that the learning curve will most probably affect the planning time without affecting treatment outcomes. 3,19,21,22,26

In the current paper, the outcomes of the first 100 patients treated at Sheba Medical Center and Assuta Medical Centers are presented, as is a discussion of the transition from intensity-modulated radiotherapy (IMRT) to volumetric modulated arc therapy (VMAT), the treatment of 2 proximal lesions with a single isocenter, treatment failures, complications, and an evaluation of the learning curves at both centers.

\section{Methods}

We retrospectively studied the records of patients treated with SRS by the first author at Sheba Medical Center and Assuta Medical Centers. After obtaining study approval from the institutional review boards, we reviewed the records of patients treated in the period from September 2011 to February 2016.

All patients were supine, immobilized in either a head to shoulders thermoplastic mask (for tumors involving the occiput to T-5) or vacuum cushions (for lesions involving T-6 to the sacrum). All patients were scanned using 1.5 -mm-thick contiguous CT slices and a large-bore CT simulator. Moreover, unless there was a contraindication, all patients underwent spine MRI with axial turbo spin echo T1-weighted sequences without and with Gd, T2-weighted sequences, and axial STIR sequences to optimize tumor delineation. Computed tomography myelography was performed in patients who could not undergo MRI or those with incomplete visualization of the neural structures or thecal sac related to an instrument artifact.

Magnetic resonance and CT image data sets were then imported into the iplannet treatment planning system (Brainlab) and rigorously fused. The clinical target vol- ume (CTV) and critical structures (cord or thecal sac in the area of the cauda equina; lungs, kidneys, bowel, and esophagus) were contoured according to the International Spine Radiosurgery Consortium consensus guidelines for target volume definition. ${ }^{8}$ Spinal cord contouring was extended $4.5 \mathrm{~mm}$ (3 CT slices) rostrally and caudally beyond the CTV in all cases. No margins were added; that is, the planning target volume (PTV) equaled the CTV. In the first 3 cases, a plan involving 7 coplanar beams using the step and shoot IMRT technique was generated with the iplannet software. The other 97 cases were planned using the Eclipse VMAT algorithm (Rapid Arc, Varian). The plan was exported from the iplannet to the Eclipse workstation. The plan was calculated using 2-3 arcs of $360^{\circ}$. Image fusion, contouring, and planning were evaluated by the planning physician (R.H.), the radiation oncologists (L.Z. and R.P.), and the physics experts (D.L., E.S., D.E., L.T., M.B.A., and D.A.). The prescription dose was 16 Gy (to cover $\geq 90 \%$ of the identified CTV) in the first 35 patients. The remaining 65 patients were treated with 18 Gy to at least $90 \%$ of the CTV. Cord constraint was set at no more than $10 \%$ of the contoured volume or absolute $0.35 \mathrm{~cm}^{3}$ to receive $10 \mathrm{~Gy}$ or above, and the maximum cord point dose (Dmax) was limited to $14 \mathrm{~Gy}$. The inverse planning algorithms used robust planning software, which accounted for instrument-related scatter and allowed for more accurate tumor and spinal cord dosing. On the day of treatment, the patient was positioned according to simulation markings. Using the ExacTrac (Brainlab) on-board imaging system, we obtained 2 diagonal stereo x-ray images and fused them to 2 digitally reconstructed radiographs generated from the simulation $\mathrm{CT}$ at the same angle. Shifts were made in the $\mathrm{x}$, $\mathrm{y}$, and $\mathrm{z}$ directions as needed, and another set of $\mathrm{x}$-ray images were obtained to confirm final patient positioning. In selected cases, on-board cone-beam CT (CBCT) was used to verify position. We found $\mathrm{CBCT}$ especially useful in the midthoracic region, as the vertebral bodies resemble each other and the ExacTrac fusion algorithm can achieve an erroneous, but still acceptable, fusion of proximal levels. Treatment was delivered using the Novalis linear accelerator with a micromultileaf collimator for beam shaping (Brainlab) at the Sheba Medical Center or the TrueBeam STx linear accelerator (Varian) at the Assuta Medical Centers. The patients were monitored during treatment by using ExacTrac (Brainlab).

For evaluation of local control, patients had undergone serial follow-up MRI examinations. Most patients had MRI studies every 3 months; those with slow-growing metastases were imaged every 4 months (sarcoma, chordoma); and those with nerve sheath tumors (NSTs) or meningiomas had serial MRI every 6 months. Images were evaluated by the first author (R.H.) and by a multidisciplinary team during the institutional tumor board and were considered to demonstrate local control if the tumor had not increased in size. Physical examination results and adverse events were recorded in the patient charts and were reported during the tumor board meetings.

\section{Training the SRS Team}

Spine radiosurgery service was initiated at the Sheba 
Medical Center in September 2011 and at the Assuta Medical Centers in September 2012. Services at both were initiated after the first author (R.H.) had undergone a 1-year fellowship at a leading SRS center, performing all stages of clinical SRS treatment and researching the field of SRS. Both radiation oncologists (L.Z. and R.P.) were experienced in brain radiosurgery and had visited centers performing spine radiosurgery. Both chief physics experts had tremendous experience with brain radiosurgery. The system vendor trained the entire team on the system before the first session. Weekly tumor boards were established, during which spine tumor patients were discussed and alternative treatments were considered. The involved physicians presented cases at local scientific meetings of neurosurgeons, spine surgeons, and oncologists. For patients referred to SRS, the SRS nurse coordinator scheduled them for the first author's clinic within a week. After the tumor board discussion, patients were referred to their insurance company for approval of the treatment. After approval, the treatment schedule was set by first determining the estimated treatment day and setting the CT and MRI dates to be 5-7 days beforehand.

\section{Results \\ Patient Population}

One hundred treatment sessions, performed at both centers (71 at Sheba and 29 at Assuta), are summarized in this paper. These treatment sessions involved 118 lesions at 179 spinal levels in 80 patients. Table 1 summarizes the demographics of and the pathological characteristics in the patients treated. In $78 \%$ of the patients, treatment was prescribed for spinal metastases. Most lesions (66\%) involved mainly the vertebral body; a smaller portion (18\%) involved the lateral or posterior aspects of the vertebra. Twelve percent consisted of intradural extramedullary tumors such as meningiomas, NSTs, and ependymomas, and only 5\% were intramedullary tumors (hemangiopericytoma, hemangioblastoma, atypical teratoid/rhabdoid tumor [ATRT]). Table 2 summarizes the major treatment values.

\section{Complications}

Complications consisted of 1 case of lumbosacral radicular pain aggravated after SRS, 3 cases of transient swallowing difficulties, and 1 case of transient nausea. Three patients had worsening of pathological fractures; 2 of them were treated with kyphoplasty, and pedicle fixation was performed in the third patient. One patient treated for C-5 thyroid carcinoma metastasis via C-5 corpectomy and instrumented fusion followed by SRS had a good primary response to treatment; however, 16 months after SRS, he developed esophageal perforation exposing the plate and a screw and required surgical intervention. Another patient, who had previously undergone surgery via a lateral transthoracic approach for T-11 metastases from renal cell carcinoma, was treated with SRS for metastases at T-4 and T-6 with a good initial response. Seven months post-SRS, the patient had esophageal bleeding requiring surgical intervention.

None of the patients developed radiation-induced my-
TABLE 1. Summary of characteristics in 100 treatment sessions

\begin{tabular}{|c|c|c|}
\hline Parameter & Absolute No. & Percentage \\
\hline $\operatorname{Sex}(M: F)$ & $56: 44$ & \\
\hline Mean patient age in yrs & 55.7 & \\
\hline Cases w/ previous radiation & 38 & \\
\hline Cases w/ previous surgery & 29 & \\
\hline \multicolumn{3}{|l|}{ Pathology (no. of cases) } \\
\hline RCC & 23 & $23 \%$ \\
\hline Sarcoma & 11 & $11 \%$ \\
\hline Colon cancer & 8 & $8 \%$ \\
\hline Breast cancer & 7 & $7 \%$ \\
\hline Nerve sheath tumor & 7 & $7 \%$ \\
\hline Lung cancer & 6 & $6 \%$ \\
\hline Prostate cancer & 5 & $5 \%$ \\
\hline Chordoma & 5 & $5 \%$ \\
\hline Melanoma & 3 & $3 \%$ \\
\hline Ewing sarcoma & 3 & $3 \%$ \\
\hline Adrenal cortical cancer & 3 & $3 \%$ \\
\hline Multiple myeloma & 2 & $2 \%$ \\
\hline Ependymoma & 2 & $2 \%$ \\
\hline Thyroid cancer & 2 & $2 \%$ \\
\hline Meningioma & 2 & $2 \%$ \\
\hline ATRT & 2 & $2 \%$ \\
\hline Hemangioblastoma & 2 & $2 \%$ \\
\hline Cholangiocarcinoma & 2 & $2 \%$ \\
\hline Malignant NST & 1 & $1 \%$ \\
\hline Hepatocellular cancer & 1 & $1 \%$ \\
\hline Gastric cancer & 1 & $1 \%$ \\
\hline Nasopharynx cancer & 1 & $1 \%$ \\
\hline Hemangiopericytoma & 1 & $1 \%$ \\
\hline \multicolumn{3}{|l|}{ Treated region (no. of cases) } \\
\hline Cervical & 21 & $21 \%$ \\
\hline Thoracic & 56 & $56 \%$ \\
\hline Lumbar & 17 & $17 \%$ \\
\hline Sacral & 6 & $6 \%$ \\
\hline Mean VAS score & 4.43 & \\
\hline \multicolumn{3}{|c|}{ Neurological status (no. of cases) } \\
\hline No deficit & 38 & $38 \%$ \\
\hline Radiculopathy & 32 & $32 \%$ \\
\hline Mild myelopathy & 18 & $18 \%$ \\
\hline Severe myelopathy & 11 & $11 \%$ \\
\hline Sphincter disability & 1 & $1 \%$ \\
\hline Lesions per session (1:2:3) & $84: 14: 2$ & \\
\hline
\end{tabular}

$\mathrm{RCC}=$ renal cell carcinoma; $\mathrm{VAS}=$ visual analog scale.

elopathy, and none were treated with steroids for more than 3 days.

\section{Local Control Rates}

Follow-up visits coupled with imaging were performed in $91 \%$ of the treated lesions. Mean follow-up was 302 
TABLE 2. Treatment characteristics

\begin{tabular}{ll}
\hline Treatment Protocol $^{*}$ & Value \\
\hline Mean prescribed dose & $17.04 \mathrm{~Gy}$ \\
\hline Mean isodose coverage & $94.20 \%$ \\
\hline Mean maximal dose & $19.76 \mathrm{~Gy}$ \\
\hline Spinal cord mean dose & $6.9 \mathrm{~Gy}$ \\
\hline Spinal cord 10 Gy & $5.68 \%$ \\
\hline Mean follow-up & 302 days \\
\hline
\end{tabular}

* Three cases of IMRT and 97 cases of VMAT.

days, and the local control rate was 95\%. Only 6 local recurrences were recorded. Three of these treatment failures occurred in patients with metastatic sarcoma (18 sarcoma lesions treated; $83 \%$ local control rate) and 2 in patients with NSTs (9 NSTs treated; 78\% local control rate). One of the NSTs was malignant and the other was a neurofibromatosis. One patient had ATRT intramedullary metastases. Two of the 6 treatment failure cases were intradural (17 cases treated; $88 \%$ local control rate), and the rest were extradural (95\% local control rate). Spinal radiosurgery was the primary treatment in 71 patients, 4 of whom had local recurrence (94\% local control rate). In contrast, 29 patients underwent surgery first, and then SRS was delivered at a later stage; 2 local recurrences were reported among these patients (93\% local control rate). Among the 6 treatment failure cases, a 16-Gy dose had been prescribed in 4 and an 18-Gy dose in the other 2. The local control rates for $16-$ Gy group and 18 -Gy group were $87 \%$ and $97 \%$, respectively, with a mean follow-up of 399 and 228 days. Among the 6 failure cases, 1 was successfully retreated with SRS, 1 with SRS and then surgery, 2 with surgery, and 2 with systemic chemotherapy since concomitant multiple metastases had been diagnosed.

\section{Multilevel Single-Session Treatment}

Sixteen patients were treated for more than 1 lesion in a single session. Of these, 9 patients harboring 2 proximal lesions were treated using a single isocenter and a single plan for both lesions with a 2-3 circumferential arcs VMAT protocol, substantially reducing the positioning and treatment time.

\section{Discussion}

Spinal radiosurgery is a multidisciplinary treatment protocol designed for the management of spinal tumors with high efficacy and low complication rates. However, this treatment has the potential for severe complication if accuracy is compromised. The procedure entails externally immobilizing the patient, imaging with multiple modalities, fusing the imaging studies and contouring the target and organs at risk, planning the treatment, assuring quality with a phantom run, and finally positioning the patient and treating with radiation. Each step holds a certain risk for errors, and each member of the team should be responsible for avoiding errors or recognizing and fixing any errors made. Any implementation of new medical services is bound to have a learning curve; SRS, a multidisciplinary endeavor, is no different. However, a PubMed search of the terms "spine radiosurgery" and "learning curve" yielded no results. Our aim in the current paper was to share the experience of our centers and allow others to better understand the complexity of building a spine radiosurgery service and avoid some of the pitfalls described above.

Pain is the main symptom of spinal tumors and a major reason for treatment. Most patients undergoing SRS suffer from cancer-related pain. ${ }^{1,6}$ Radiosurgical treatment is performed on nonsedated patients and necessitates a motionless supine position for the entire duration of treatment. Obviously, a patient in extreme pain cannot fulfill these requirements. Various SRS technologies allow for a reduction in treatment duration, enabling the treatment of patients in pain. The routine method for treatment planning and delivery is IMRT. Multiple (usually static) beams of radiation are directed from 7 to 10 different angles. Volumetric modulated arc therapy or RapidArc (Eclipse, Varian) is a radiation planning and delivery method combining dynamic arcs and IMRT and is currently used by us. We have found VMAT planning and treatment for SRS to be superior to IMRT not only in terms of shorter delivery times but also in terms of conformality, homogeneity, and safety profile. ${ }^{27}$

Although a decline in complication rates in accordance with a learning curve was not evident in our current study, the complication rate was low and comparable with that in the published literature. Our rate may be attributable to the teams at both centers, which are highly experienced in brain radiosurgery and have already established the requisite teamwork.

As mentioned previously, the prescribed radiation dose was increased after the first 35 treatment sessions, which increased the local control rate from $89 \%$ to $97 \%$. This change was supported by both the literature, which demonstrated a higher local control rate with higher doses, and the confidence level of the team, which allowed safe treatment with higher doses. ${ }^{9,17,18}$

New radiotherapy tools such as VMAT to replace the standard IMRT were adopted with caution. During the transition 10 cases were planned using both IMRT and VMAT, and plan conformity, compliance with organ-atrisk constraints, and treatment time were compared and found to be superior with VMAT. ${ }^{27}$ Only later was VMAT defined as the default planning and treatment method.

While brain radiosurgery is well established and easily compensated by insurance companies, a great deal of effort was made in establishing patient referral sources and assuring the insurance companies that this costly, highprecision treatment would be saved for highly selected patients (radioresistant tumors, oligometastatic disease, standard radiotherapy failures).

Spinal radiosurgery is a safe treatment with high local control rates, quick and efficacious pain control, and low complication rates. ${ }^{1,11,14}$ In the present study, the local control rates and low complication rate were congruent with those previously reported. Radioresistant tumors have a less favorable local control rate. ${ }^{2}$ Miller et al. ${ }^{20}$ demonstrated lower local control rates for a single fraction of 16 Gy for metastatic sarcoma treatment; hence, they recom- 
mended treatment with $18 \mathrm{~Gy}$ for these pathologies. Of the 3 sarcoma patients with recurrences in the present study, 2 had been treated with a prescribed dose of $16 \mathrm{~Gy}$ and only 1 had been treated with $18 \mathrm{~Gy}$. Of the 2 NST patients, both had been treated with $16 \mathrm{~Gy}$. Four (67\%) of the 6 failure lesions were treated with $16 \mathrm{~Gy}$, a dose that was amplified to 18 Gy by our team more than 2 years ago for all pathologies. Radiation-induced myelopathy has not occurred even after increasing the prescribed dose.

Treatment of proximal lesions using a single-isocenter, single-plan method was initiated as soon as the VMAT protocol became routine. In each of these cases, a plan was executed and carefully reviewed for maximal coverage, best homogeneity, and minimal dose to the bridging structures. The obvious advantages of reducing treatment time in terms of both patient positioning and treatment duration with no interference in dose delivery and coverage led to our adoption of this technique.

\section{Study Limitations}

This retrospective study was performed by the treating physician; hence, bias cannot be eliminated. As SRS is a very safe and effective therapy, assessing the learning curve of a single treatment center can be very challenging. Since the group treated to the $18-G y$ prescribed dose was the later-treated group, its follow-up time is shorter than that for the 16-Gy group; hence, local recurrence comparisons between these groups are biased. Pain scores were not routinely recorded for patients and thus were not reported here.

\section{Conclusions}

Spinal radiosurgery is a safe and effective treatment for spinal tumors with high rates of local control, and higher radiation doses result in superior local control rates. Radioresistant tumors should be treated with the highest possible dose. The VMAT technology allows for shorter and more conformal delivery, thus reducing patient movement. Radiosurgery necessitates a multidisciplinary team approach, and the entire team must be properly trained to limit the effects of the learning curve on outcome.

\section{References}

1. Angelov L, Chao S, Heng DY, Djemil T, Kolar M, Suh J: Stereotactic spine radiosurgery (SRS) for pain and tumor control in patients with spinal metastases from renal cell carcinoma: a prospective study. Int J Radiat Oncol Biol Phys 72 (1 Suppl):S489, 2008 (Abstract \#2716)

2. Balagamwala EH, Angelov L, Koyfman SA, Suh JH, Reddy CA, Djemil T, et al: Single-fraction stereotactic body radiotherapy for spinal metastases from renal cell carcinoma. J Neurosurg Spine 17:556-564, 2012

3. Benzel EC, Orr RD: A steep learning curve is a good thing! Spine J 11:131-132, 2011

4. Benzil DL, Saboori M, Mogilner AY, Rocchio R, Moorthy CR: Safety and efficacy of stereotactic radiosurgery for tumors of the spine. J Neurosurg 101 (Suppl 3):413-418, 2004

5. Bilsky MH, Yamada Y, Yenice KM, Lovelock M, Hunt M,
Gutin PH, et al: Intensity-modulated stereotactic radiotherapy of paraspinal tumors: a preliminary report. Neurosurgery 54:823-831, 2004

6. Cole JS, Patchell RA: Metastatic epidural spinal cord compression. Lancet Neurol 7:459-466, 2008

7. Coleman MP, Forman D, Bryant H, Butler J, Rachet B, Maringe C, et al: Cancer survival in Australia, Canada, Denmark, Norway, Sweden, and the UK, 1995-2007 (the International Cancer Benchmarking Partnership): an analysis of population-based cancer registry data. Lancet 377:127-138, 2011

8. Cox BW, Spratt DE, Lovelock M, Bilsky MH, Lis E, Ryu S, et al: International Spine Radiosurgery Consortium consensus guidelines for target volume definition in spinal stereotactic radiosurgery. Int J Radiat Oncol Biol Phys 83:e597e605, 2012

9. Damast S, Wright J, Bilsky M, Hsu M, Zhang Z, Lovelock $\mathrm{M}$, et al: Impact of dose on local failure rates after imageguided reirradiation of recurrent paraspinal metastases. Int J Radiat Oncol Biol Phys 81:819-826, 2011

10. Gerszten PC, Burton SA, Ozhasoglu C, Vogel WJ, Welch WC, Baar J, et al: Stereotactic radiosurgery for spinal metastases from renal cell carcinoma. J Neurosurg Spine 3:288-295, 2005

11. Gerszten PC, Burton SA, Ozhasoglu C, Welch WC: Radiosurgery for spinal metastases: clinical experience in 500 cases from a single institution. Spine (Phila Pa 1976) 32:193-199, 2007

12. Gerszten PC, Burton SA, Welch WC, Brufsky AM, Lembersky BC, Ozhasoglu C, et al: Single-fraction radiosurgery for the treatment of spinal breast metastases. Cancer 104:22442254,2005

13. Gerszten PC, Welch WC: Cyberknife radiosurgery for metastatic spine tumors. Neurosurg Clin N Am 15:491-501, 2004

14. Hall WA, Stapleford LJ, Hadjipanayis CG, Curran WJ, Crocker I, Shu HK: Stereotactic body radiosurgery for spinal metastatic disease: an evidence-based review. Int J Surg Oncol 2011:979214, 2011

15. Harel R, Angelov L: Spine metastases: current treatments and future directions. Eur J Cancer 46:2696-2707, 2010

16. Harel R, Emch T, Chao S, Elson P, Krishnaney A, Djemil $\mathrm{T}$, et al: Quantitative evaluation of local control and wound healing following surgery and stereotactic spine radiosurgery for spine tumors. World Neurosurg 87:48-54, 2016

17. Laufer I, Iorgulescu JB, Chapman T, Lis E, Shi W, Zhang $Z$, et al: Local disease control for spinal metastases following "separation surgery" and adjuvant hypofractionated or high-dose single-fraction stereotactic radiosurgery: outcome analysis in 186 patients. J Neurosurg Spine 18:207-214, 2013

18. Lovelock DM, Zhang Z, Jackson A, Keam J, Bekelman J, Bilsky M, et al: Correlation of local failure with measures of dose insufficiency in the high-dose single-fraction treatment of bony metastases. Int J Radiat Oncol Biol Phys 77:12821287,2010

19. McLoughlin GS, Fourney DR: The learning curve of minimally-invasive lumbar microdiscectomy. Can J Neurol Sci 35:75-78, 2008

20. Miller JA, Balagamwala EH, Angelov L, Suh JH, Djemil T, Magnelli A, et al: Stereotactic radiosurgery for the treatment of primary and metastatic spinal sarcomas. Technol Cancer Res Treat [epub ahead of print], 2016

21. Nowitzke AM: Assessment of the learning curve for lumbar microendoscopic discectomy. Neurosurgery 56:755-762, 2005

22. O'Malley BW Jr, Grady MS, Gabel BC, Cohen MA, Heuer GG, Pisapia J, et al: Comparison of endoscopic and microscopic removal of pituitary adenomas: single-surgeon experi- 
ence and the learning curve. Neurosurg Focus 25(6):E10, 2008

23. Patchell RA, Tibbs PA, Regine WF, Payne R, Saris S, Kryscio RJ, et al: Direct decompressive surgical resection in the treatment of spinal cord compression caused by metastatic cancer: a randomised trial. Lancet 366:643-648, 2005

24. Quaresma M, Coleman MP, Rachet B: 40-year trends in an index of survival for all cancers combined and survival adjusted for age and sex for each cancer in England and Wales, 1971-2011: a population-based study. Lancet 385:12061218,2015

25. Quraishi NA, Gokaslan ZL, Boriani S: The surgical management of metastatic epidural compression of the spinal cord. J Bone Joint Surg Br 92:1054-1060, 2010

26. Rong LM, Xie PG, Shi DH, Dong JW, Liu B, Feng F, et al: Spinal surgeons' learning curve for lumbar microendoscopic discectomy: a prospective study of our first 50 and latest 10 cases. Chin Med J (Engl) 121:2148-2151, 2008

27. Zach L, Tsvang L, Alezra D, Ben Ayun M, Harel R: Volumetric modulated arc therapy for spine radiosurgery: superior treatment planning and delivery compared to static beam intensity modulated radiotherapy. BioMed Res Int 2016:6805979, 2016

\section{Disclosures}

Dr. Zach holds a patent and has 2 patents pending with Brainlab.

\section{Author Contributions}

Conception and design: Harel, Zach. Acquisition of data: Harel. Analysis and interpretation of data: Harel. Drafting the article: Harel. Critically revising the article: Harel, Pfeffer, Levin. Reviewed submitted version of manuscript: Harel. Approved the final version of the manuscript on behalf of all authors: Harel. Administrative/technical/material support: Levin, Shekel, Epstein, Tsvang, Ben-Ayun, Alezra. Study supervision: Pfeffer.

\section{Correspondence}

Ran Harel, Department of Neurosurgery, Sheba Medical Center, Ramat Gan, Israel. email: ran.harel@sheba.health.gov.il. 